\title{
PREFACE: SPECIAL ISSUE “COMPLEX FLUIDS AND HEAT TRANSFER"
}

Complex fluids and heat transfer are among the crucial issues in a number of industrial applications, such as thermal and power engineering, energy conversion and utilization, energy storage, intelligent materials, chemical industry, biological engineering, etc. In these industrial applications, the use of complex fluids, such as non-Newtonian fluids, surfactant fluids, nanofluids, magnetic fluids, self-rewetting fluids, etc., is often a way to increase productivity not only in the process itself, but also to take advantage of various subsequent phenomena. For example, various special phenomena appear in the heat transfer process with complex fluids, such as Marangoni convection, thermocapillary flows, interfacial instability, and the Leidenfrost effect. All these phenomena play key roles in enhancing heat transfer. However, the mechanism underlying them is very complex. In addition, the physical properties of complex fluids, such as viscosity, wettability, and surface tension, change in comparison to those of normal fluids. They also influence the heat transfer performance significantly. Hence, it is necessary and urgent to do research on these special issues appearing simultaneously or separately during heat transfer processes with complex fluids. Fortunately, these issues have been the focus of attention of a large number of researchers in the areas of applied mathematics, fluid dynamics, energy, and chemical engineering. These scientists and engineers have made great efforts to study, analyze, and overcome the problems found in their research. Meanwhile, the experiments, simulations, and modeling done to quantitatively characterize the results have become standard methods to explore new mechanisms in complex fluids and heat transfer. Many meaningful results have been obtained.

In order to present the advances in the area, a Special Issue on "Complex Fluids and Heat Transfer" is published in the present issue of the journal Interfacial Phenomena and Heat Transfer (IPHT). This Special Issue involves both experimental and numerical studies on complex fluids and heat transfer. The research topics cover the following five scientific directions: (1) boiling, nucleation, bubbles, entrainment, critical heat flux; (2) two-phase flows, heat and mass transfer in microchannels and ministructues; (3) nano- and microstructured surfaces for enhancement of phase change phenomena; (4) thermocapillary flows, instability, interfacial waves, evaporation, and condensation; and (5) contact line phenomena, droplets, spray, film flows, dry spot formation, and wettability effects.

According to the requirements of IPHT, all the papers published in this Special Issue have undergone a rigorous peer-review process. We would like to thank all authors and reviewers for their important contributions to the publication of the final form of this Special Issue. It is our hope that this issue will provide a resource and inspiration for future work regarding heat transfer enhancement techniques with complex fluids.

\section{Guest Editors:}

Jin-Jia Wei

Xi' an Jiaotong University, China

Bo-Feng Bai

Xi' an Jiaotong University, China

Vladimir S. Ajaev

Southern Methodist University, USA

Luis A. Dávalos-Orozco

Universidad Nacional Autónoma de México, México 